\title{
Peuplement avifaunique du Campus Universitaire Jean Lorougnon Guédé, Daloa et sa périphérie (Centre-Ouest de la Côte d'Ivoire)
}

\author{
Gnininté Maxime ZEAN*, Dibié Bernard AHON et Béné Jean-Claude KOFFI
}

\author{
Laboratoire Biodiversité et Gestion Durable des Ecosystèmes Tropicaux (BioEcoTrop), UFR \\ Environnement, Université Jean Lorougnon Guédé, Côte d'Ivoire. \\ *Auteur correspondant; E-mail: maximezean@gmail.com; Tél: (00225) 59083715
}

\section{RESUME}

En Côte d'Ivoire, l'essentiel des études ornithologiques a été effectué dans les domaines classés de l'Etat au détriment des milieux urbains qui constituent cependant des écosystèmes particuliers. Pour pallier ce déficit d'informations, des études ont été réalisées sur le Campus Universitaire Jean Lorougnon Guédé (CUJLoG) et sa périphérie. La méthodologie était basée sur des points d'écoute, de repasse de vocalisations, de capture et recapture au filet japonais et de comptages à durée déterminée sur des transects linéaires. Au total, 105 espèces reparties en 43 familles et 17 ordres ont été inventoriées. Une de ces espèces (Circus macrourus) est quasi-menacée, neuf sont caractéristiques du biome des forêts guinéo-congolaises et une de la savane soudano-guinéenne. Ce peuplement est caractérisé par 05,71\% d'espèces dominantes ; 08,57\% d'espèces régulières ; 41,90\% d'espèces rares et 43,81\% d'espèces accidentelles. Aussi, 14,28\% est totalement migratrice ; $21,90 \%$ est partiellement migratrice et $63,82 \%$ est résidente $(\mathrm{R})$. En outre, $68,57 \%$ a une préférence pour les milieux ouverts (f) $; 15,24 \%$ pour les milieux humides (Ea) et $16,18 \%$ pour les forêts $(\mathrm{F})$. Avec cette avifaune riche et diversifiée, le CUJLoG pourrait être une Zone d'Importance pour la Conservation des Oiseaux.

(C) 2018 International Formulae Group. All rights reserved.

Mots clés: Peuplement, avifaune, Université Jean Lorougnon Guédé, Côte d'Ivoire.

\begin{abstract}
In Côte d'Ivoire, most ornithological studies have been carried out in the State's classified areas to the detriment of urban environments, which nevertheless constitute particular ecosystems. To compensate this information deficit, studies were carried out on the Jean Lorougnon Guédé University Campus (CUJLoG) and its periphery. The methodology was based on listening points, callplayback, capture and recapture with the Japanese net and fixed duration counts on linear transects. In total, 105 species divided into 43 families and 17 orders were inventoried. One of these species (Circus macrourus) is almost threatened, nine are characteristic of the Guineo-Congolese forest biome and one of the Sudano-Guinean savannah. This stand is characterized by $05.71 \%$ dominant species; $08.57 \%$ regular species; $41.90 \%$ rare species and $43.81 \%$ accidental species. Also, $14.28 \%$ is fully migratory; $21.90 \%$ is partially migratory and $63.82 \%$ is resident (R). In addition, $68.57 \%$ have a preference for open environments (f); $15.24 \%$ for wetlands (Ea) and $16.18 \%$ for forests $(\mathrm{F})$. With this rich and diverse birdlife, CUJLoG could be an Important Bird Area.
\end{abstract}

(C) 2018 International Formulae Group. All rights reserved.

Keywords: Settlement, avifauna, Jean Lorougnon Guédé University, Ivory Coast. 


\section{INTRODUCTION}

La diminution progressive de la biodiversité est une préoccupation mondiale. La conférence sur la biodiversité qui s'est tenue à Rio de Janeiro en 1992 et les nombreuses recommandations qui ont été faites à cette occasion, ont montré l'importance de la biodiversité sur le plan génétique, social, scientifique, éducatif, culturel et environnemental (Goudard, 2007 ; Zhang et al., 2007; Le Roux et al., 2008). Cette préoccupation autour de la biodiversité témoigne d'une prise de conscience de la dégradation des écosystèmes en vue d'une gestion durable (Le Roux et al., 2008). Les causes de cette dégradation sont attribuées aux activités humaines, en particulier l'agriculture et l'urbanisation, qui conduisent à la modification souvent irréversibles d'une grande partie des habitats naturels des oiseaux (Bot et al., 2000). L'urbanisation est une manifestation concrète de la perte d'habitat. Elle est un phénomène mondial qui touche de nombreux points chauds de la biodiversité. Au cours des dernières années, le processus s'est amplifié. En effet, partout dans le monde, les humains se concentrent de plus en plus dans les villes (Cincotta, 2000). Pourtant, il est bien établi que l'urbanisation permet une augmentation de la densité des populations d'oiseaux (Chace et Walsh, 2006). Elle a pour conséquence de favoriser les espèces résidentes au détriment des espèces migratrices et certaines guildes alimentaires au détriment d'autres (Huillet, 2007). Par ailleurs, la biodiversité avifaunique est connue pour son rôle bio-indicateur aux changements de l'environnement et pour sa capacité de colonisation d'une grande palette de milieux différents (Yaokokoré-Béibro, 2010).

En Côte d'Ivoire, les études ornithologiques menées essentiellement dans les domaines classés de l'Etat (parcs nationaux et réserves naturelles, dans les forêts classées et les jardins biologiques) ont permis de recenser 758 espèces d'oiseaux (Ahon, 2016). Cependant, l'avifaune reste encore mal connue dans la plupart des zones urbaines. Les quelques données avifauniques des milieux urbains de Côte d'Ivoire sont toutes issues des travaux de Konan et al., (2015) et de Yaokokoré-Béibro et al., (2015a ; 2015b). Or, la plupart des villes ivoiriennes ont en leur sein plusieurs types d'habitats notamment les jachères, les zones humides, les forêts, les zones de cultures, etc. Chaque type d'habitats ayant sa faune aviaire spécifique (Yaokokoré-Béibro et al., 2015b, 2015c), il apparaît donc nécessaire de conduire une étude ornithologique dans un milieu urbain avec plusieurs types d'habitats. Le choix a été porté sur le CUJLoG et sa périphérie où il n'existe aucune donnée avifaunique avant la présente étude qui a pour objectif de contribuer à une meilleure connaissance de l'avifaune urbaine de la Côte d'Ivoire. De manière spécifique, il s'agira de connaître et de caractériser le peuplement avifaunique du CUJLoG et de sa périphérie.

\section{MATERIEL ET METHODES Site d'étude}

Cette étude s'est déroulée dans le domaine du Campus universitaire Jean Lorougnon Guédé (d'une superficie de 400 ha) et dans sa zone périphérique. Il est situé au nord de la ville de Daloa plus précisément au quartier Tazibouo (Figure 1). La ville de Daloa, chef-lieu du département et de la région du Haut-Sassandra, est située au Centre-Ouest de la Côte d'Ivoire entre $6^{\circ} 53^{\prime}$ de latitude Nord et $6^{\circ} 27^{\prime}$ de longitude Ouest (MPD, 2015). La région du Haut Sassandra est marquée par un climat de type subéquatorial à deux saisons dont une saison de pluie (mars à octobre) et une saison sèche (novembre à février). Les températures moyennes mensuelles oscillent entre $24^{\circ}$ et 28 ${ }^{\circ} \mathrm{C}$ avec le mois de février $\left(\mathrm{T}=28^{\circ} \mathrm{C}\right)$ comme étant le mois le plus chaud. Quant aux précipitations moyennes mensuelles, elles varient entre $6 \mathrm{~mm}$ et $104 \mathrm{~mm}$ de pluie dont le pic est atteint dans le mois d'août (Kouamé et al., 1998). La végétation est de type forestier au sud et de type savanicole arboré au nord (Kouakou et al., 2005). Des données relatives à la faune sauvage mammalienne sont disponibles (Bamba, 2015 ; Kouakou et al., 2017).

\section{Choix des sites et collectes des données}

L'étude s'est déroulée entre le 16 février et le 15 avril 2017 (en fin de saison sèche et en début de la saison pluvieuse) à travers des observations diurnes et nocturnes. Le CUJLoG a été subdivisé en quatre zones d'étude en plus de sa zone périphérique représentée par une zone humide. Cette subdivision a été faite sur la base de la présence ou l'absence de zones humides, de 
l'hétérogénéité de la végétation et du degré d'anthropisation (l'intensité ou l'ampleur des activités humaines) du milieu. Ainsi, cinq zones (la zone dégradée, la zone de forêt secondaire, la zone bâtie, la zone de carrière et la zone humide) ont été identifiées (Figure 2). Dans chaque zone d'étude, il a été réalisé un transect linéaire de $1 \mathrm{~km}$ le long duquel cinq stations d'écoute (équidistantes de 250 mètres les unes des autres) y ont été repérées à l'aide d'un GPS (Garmin 60 CSx). Trois différentes méthodologies ont été appliquées pour chacune des zones d'étude.

En effet, les observations diurnes (de $06 \mathrm{~h} 30 \mathrm{mn}$ à $18 \mathrm{~h} 30 \mathrm{mn}$ ), ont été réalisées sur chaque itinéraire échantillon de chaque zone. La marche lente et silencieuse le long de cet itinéraire échantillon avec arrêt de $5 \mathrm{mn}$ à chaque station d'écoute, a constitué la principale méthodologie. Les inventaires étaient basés sur le décompte systématique et exclusif de tous les oiseaux vus ou entendus le long des cinq transects. A chaque station, le ciel a été régulièrement scruté à l'aide d'une paire de jumelles (Bushnell 10 x $50 \mathrm{~mm}$ ) afin d'identifier grâce aux observations visuelles, les espèces d'oiseaux qui survolent le site. Les cimes, les branchages et les feuillages des grands arbres ont été fréquemment balayés à l'aide d'un télescope (Ojoticron ES 80 GA SD). Ces parcours ont été faits à pieds à deux reprises en aller et retour le matin ainsi que l'après-midi (soit $4 \mathrm{~km} /$ jour) par beau temps (ciel non couvert, absence de pluie et de grand vent, bonne visibilité, etc.). Ces observations ont eu lieu aux heures où la majorité des espèces diurnes est active, c'est-à-dire, le matin entre $06 \mathrm{~h} 30 \mathrm{mn}$ et $10 \mathrm{~h} 30 \mathrm{mn}$ et dans l'après-midi entre $14 \mathrm{~h} 30 \mathrm{mn}$ et $18 \mathrm{~h} 30 \mathrm{mn}$, soit $8 \mathrm{~h}$ d'observation journalière effective sur chaque transect. Ces horaires correspondent aussi à une période d'activité suffisamment hétérogène chez les oiseaux diurnes (Bibby et al., 1992 ; Yaokokoré-Béibro, 2001 ; Gibbons et Gregory, 2006). Ce même protocole de la marche lente et silencieuse a été effectué à trois reprises (soit 3 journées successives par itinéraire échantillon). Au total $60 \mathrm{~km}$ ont été parcourus en $120 \mathrm{~h}$ d'observation, réparties en 15 journées. Ainsi, pour chaque espèce donnée, n'a-t-il été retenu que le plus grand nombre d'individus observés lors du parcours du transect en aller et retour afin de prendre en compte tous les individus vus ou entendus lors des parcours tout en évitant de compter doublement certains individus. En effet, certains individus observés en aller sur ce parcours peuvent passer inaperçus au retour (et vice-versa) et d'autres individus peuvent être observés aussi bien en aller qu'au retour $\mathrm{du}$ parcours. En plus de cette principale méthodologie, la méthode des points fixes d'écoute et celle de capture et recapture aux filets japonais ont été utilisées. La méthode dénommée "points fixes d'observation (hors transect)» a été utilisée pendant toute une journée entre 06 h $30 \mathrm{mn}$ et 18 h $30 \mathrm{mn}$ (soit $12 \mathrm{~h}$ d'observation journalière par zone d'étude). Ainsi, pour l'ensemble des cinq zones d'étude, $60 \mathrm{~h}$ d'observation ont été consacrées à cette méthode en cinq journées. Quant à celle de la capture et recapture aux filets japonais de $12 \mathrm{~m}(12 \times 2,5 \mathrm{~m}, 16 \mathrm{~mm}$ de mailles), il y a été consacré $12 \mathrm{~h}$ d'observation (de 06 h $30 \mathrm{mn}$ à 18 h $30 \mathrm{mn}$ dans la seule zone dite de forêt secondaire susceptible d'abriter des espèces d'oiseaux peu mélodieuses qui peuvent passer inaperçues à cause de l'habitat très dense de cet type de milieu) au point de coordonnées géoréférentielles $\left.06^{\circ} 54,746^{\prime} \mathrm{N} / 06^{\circ} 54,746^{\circ} \mathrm{O}\right)$. $\mathrm{Au}$ total, $192 \mathrm{~h}$ d'observations effectives réparties en 21 journées ont été accordées à l'inventaire d'espèces d'oiseaux diurnes.

Pour les observations nocturnes, les inventaires ont été effectués sur les itinéraires échantillons utilisés pour l'échantillonnage des espèces diurnes. Trois stations d'écoute (distantes de 0,5 km les unes des autres) ont été réalisées sur chaque transect de $1 \mathrm{~km}$. La technique de repasse de vocalisations a constitué la principale méthodologie. Les chants de toutes les espèces d'oiseaux nocturnes telles que les Engoulevents, quelques vanneaux et les oiseaux de proie susceptibles d'être rencontrées dans la zone ont été passés en revue (10 au total). Les chants des oiseaux sont issus de la collection de Chappuis, 2000. Ces chants ont été diffusés grâce à un lecteur de disques compacts (Marquant MDM-14 et des baffles (SONY)). A chaque station, il a été passé en revue les vocalisations de ces dix espèces (de la plus petite à la plus grande espèce, avec bien sûr les chants des oiseaux de proie en dernière position) et des notes ont été prises sur les espèces entendues ou vues grâce à une grande lampe de poche de marque GARRITY. La vocalisation d'une espèce donnée était écoutée pendant $1 \mathrm{mn}$, suivie de $1 \mathrm{mn}$ d'attente avant 
de passer à une autre espèce. Les observations ont été faites en pleine lune, de $19 \mathrm{~h} 00$ à $21 \mathrm{~h}$ 00 en aller et de 04 h 00 à 06 h 00 du matin pour le chemin retour (soit $2 \mathrm{~km} /$ nuit et $4 \mathrm{~h}$ d'observation / nuit). Ainsi, deux nuits d'observation ont été consacrées à chacune des cinq zones d'étude. Ce qui correspond à $40 \mathrm{~h}$ d'observations nocturnes effectives sur le terrain, reparties en 10 nuits et $20 \mathrm{~km}$ parcourus.

L'inventaire de l'avifaune (diurne et nocturne) du CUJLoG a donc été réalisé en $232 \mathrm{~h}$ réparties en 21 journées et 10 nuits.

\section{Analyse des données}

Les données recueillies sur le terrain ont permis de calculer (à l'aide du tableur Excel et $\mathrm{R}$ version 2.8.0) les différents paramètres liés à la notion de fréquence, d'abondance et de diversité. Pour chacune des espèces recensées, ont été indiqués l'habitat préférentiel et le statut migratoire ou biogéographique (Borrow et Demey, 2001), le statut de conservation (UICN, 2018) et le biome (Stattersfield et al., 1998 ; Fishpool et Evans, 2001). L'indice de dominance a été également mentionné selon la valeur de la Fréquence relative (Fr) définie par Thiollay (1986). Pour cet auteur si Fr > 5\%, l'espèce est dite dominante ; si $1 \% \leq \mathrm{Fr} \leq 5 \%$, elle est dite régulière ; si $0,2 \% \leq \mathrm{Fr} \leq 1 \%$, elle est dite rare et si $\mathrm{Fr}<0,2 \%$, elle est dite accidentelle. Il convient de signaler que dans un souci de standardisation des noms des oiseaux consignés dans les différents tableaux, il a été fait référence à la nomenclature, la taxinomie et l'ordre de Borrow et Demey (2001). Les noms scientifiques, les genres et les ordres de certaines espèces d'oiseaux ont été corrigés selon Lepage (2018).

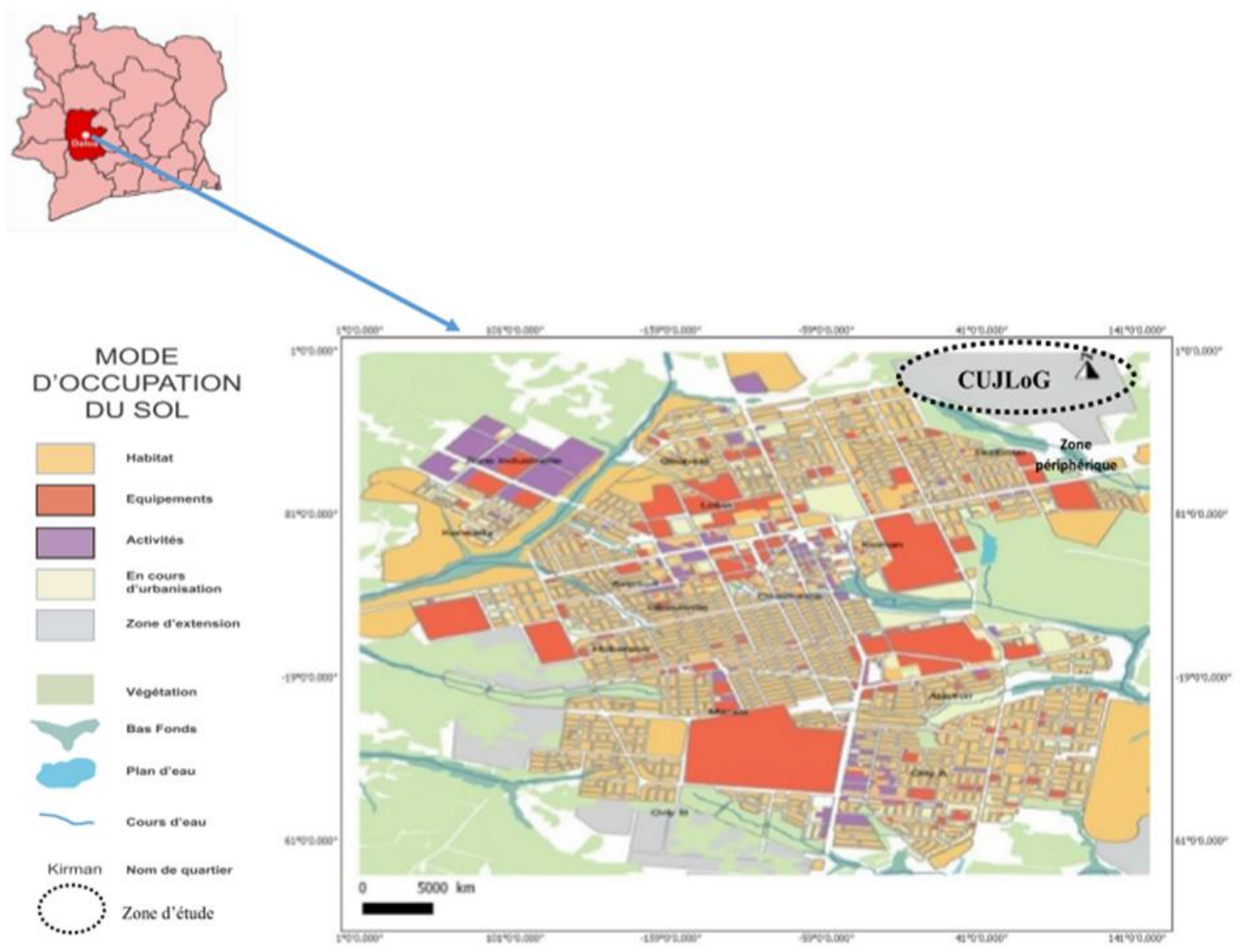

Figure 1: Carte de la ville de Daloa indiquant la localisation du Campus Universitaire Jean Lorougnon Guédé (BNETD, 2007 puis modifié). 

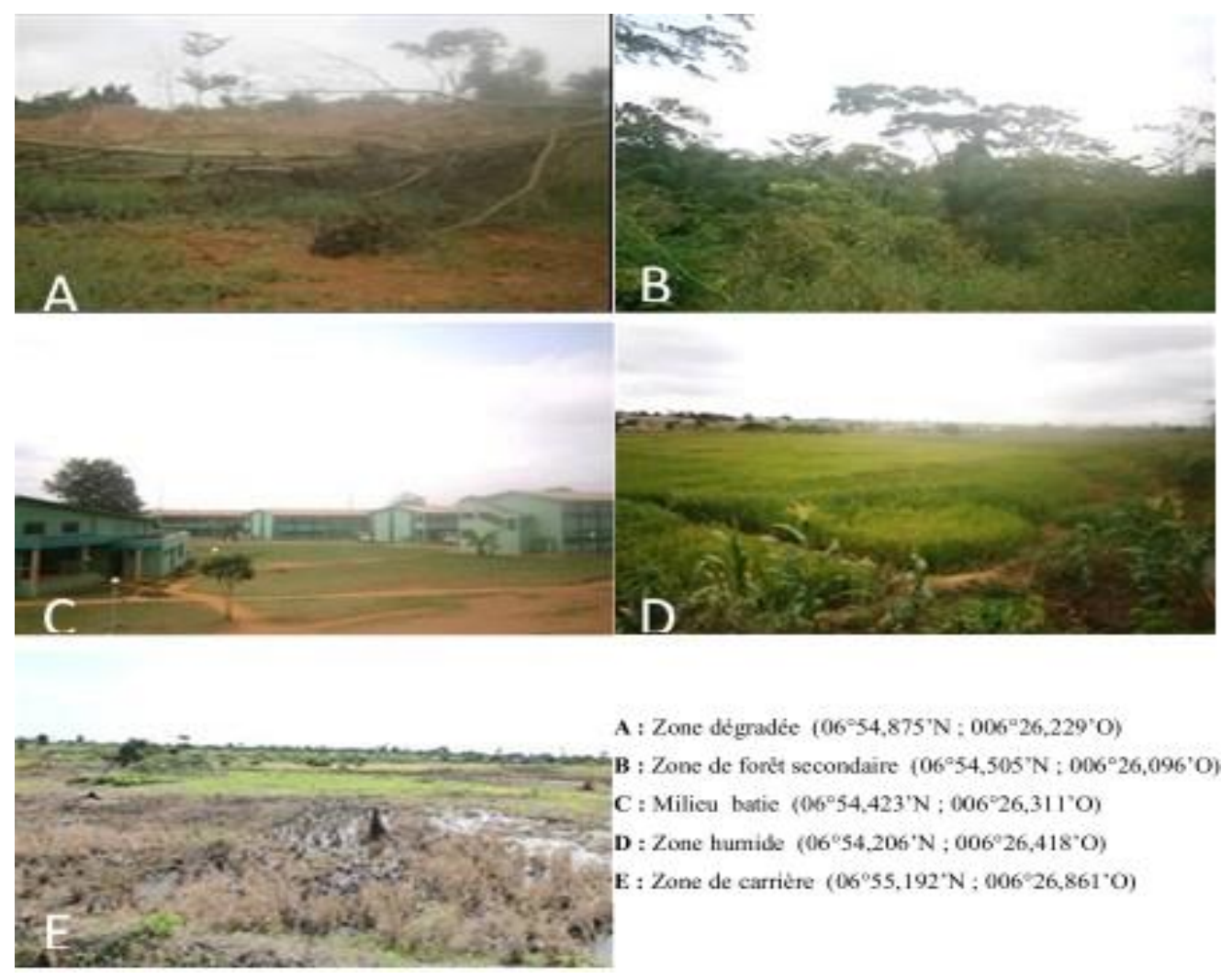

Figure 2: Vues partielles des zones d'échantillonnage de l'avifaune du CUJLoG et de sa périphérie (Photos Ahon).

\section{RESULTATS}

\section{Diversité et abondance de l'avifaune du CUJLoG}

L'ensemble des habitats inventoriés dans cette étude a permis de recenser 105 espèces d'oiseaux avec un effectif global de 2413 individus, répartis entre 43 familles et 17 ordres (Tableau 1) parmi lesquelles, une espèce bénéficie d'une protection d'intérêt mondial. Il s'agit du Busard pâle Circus macrourus de la catégorie Quasi-menacée (NT). Les autres espèces appartiennent à la catégorie Préoccupation mineure (LC). La Figure 3 illustre les photographies de quelques espèces d'oiseaux observées dans la zone d'étude.

L'analyse des résultats montre que les familles les plus représentées du point de vue de la richesse spécifique sont celles des Accipitridae, des Alcedinidae, des Ardeidae, des Columbidae, des Cuculidae, des Hirundinidae et des Cisticolidae avec six espèces chacune. Ces sept principales familles renferment $40 \%$ du nombre d'espèces d'oiseaux de la zone d'étude. En termes d'individus des différentes espèces, les six espèces principales sont par ordre de fréquence et d'abondance décroissante : le Capucin nonnette Spermestes cucullatus ( $\mathrm{Fr}=18,20 \%$; 439 individus) ; le Tisserin gendarme Ploceus cucullatus $(\mathrm{Fr}=$ $10,15 \% ; 245$ individus) ; la Tourterelle à collier Streptopelia semitorquata $(\mathrm{Fr}=$ 8,79\%; 212 individus) ; le Guêpier à gorge blanche Merops albicollis $(\mathrm{Fr}=8 \% ; 193$ individus) ; 1'Aigrette intermédiaire Egretta intermedia $(\mathrm{Fr}=7,54 \% ; 182$ individus) et le Moineau gris Passer griseus $(\mathrm{Fr}=7,21 \%$; 174 individus). Les plus grandes valeurs en termes de richesse spécifique (65 espèces) et d'indice de diversité $\left(H^{\prime}=3,52\right)$ ont été observées dans la zone de carrière. Par contre, les plus faibles valeurs ont été observées dans la zone bâtie et sont respectivement de 18 espèces et 2,32 comme indice de diversité. 
Caractéristiques du peuplement avifaunique du CUJLoG

Le peuplement d'oiseaux de la zone d'étude est caractérisé par $05,71 \%$ d'espèces dominantes, $08,57 \%$ d'espèces régulières, $41,90 \%$ d'espèces rares et $43,81 \%$ d'espèces accidentelles. Neuf $(8,57 \%)$ des espèces recensées (Ceyx lecontei ; Alcedo leucogaster ; Eurystomus gularis ; Tockus fasciatus ; Psalidoprocne nitens ; Psalidoprocne obscura ; Sylvietta virens, Hylia prasina et Ploceus nigerrimus) sont endémiques du biome des forêts guinéo-congolaises (A05) et une espèce caractéristique de la savane soudano- guinéenne (A04). A partir des statuts biogéographiques des différentes espèces (Tableau 1), il ressort que $14,28 \%$ des espèces recensées sont totalement migratrices $(04,76 \%$ d'espèces migratrices intra-africaines $(M)$ et $09,52 \%$ d'espèces migratrices du paléarctiques $(\mathrm{P})), 21,90 \%$ de ces espèces sont partiellement migratrices et $63,82 \%$ sont résidentes (R). En termes d'habitats, $68,57 \%$ des espèces ont une préférence pour les milieux ouverts (f), $15,24 \%$ des espèces pour les milieux humides $(\mathrm{Ea})$ et $16,18 \%$ des espèces pour les forêts $(\mathrm{F})$.
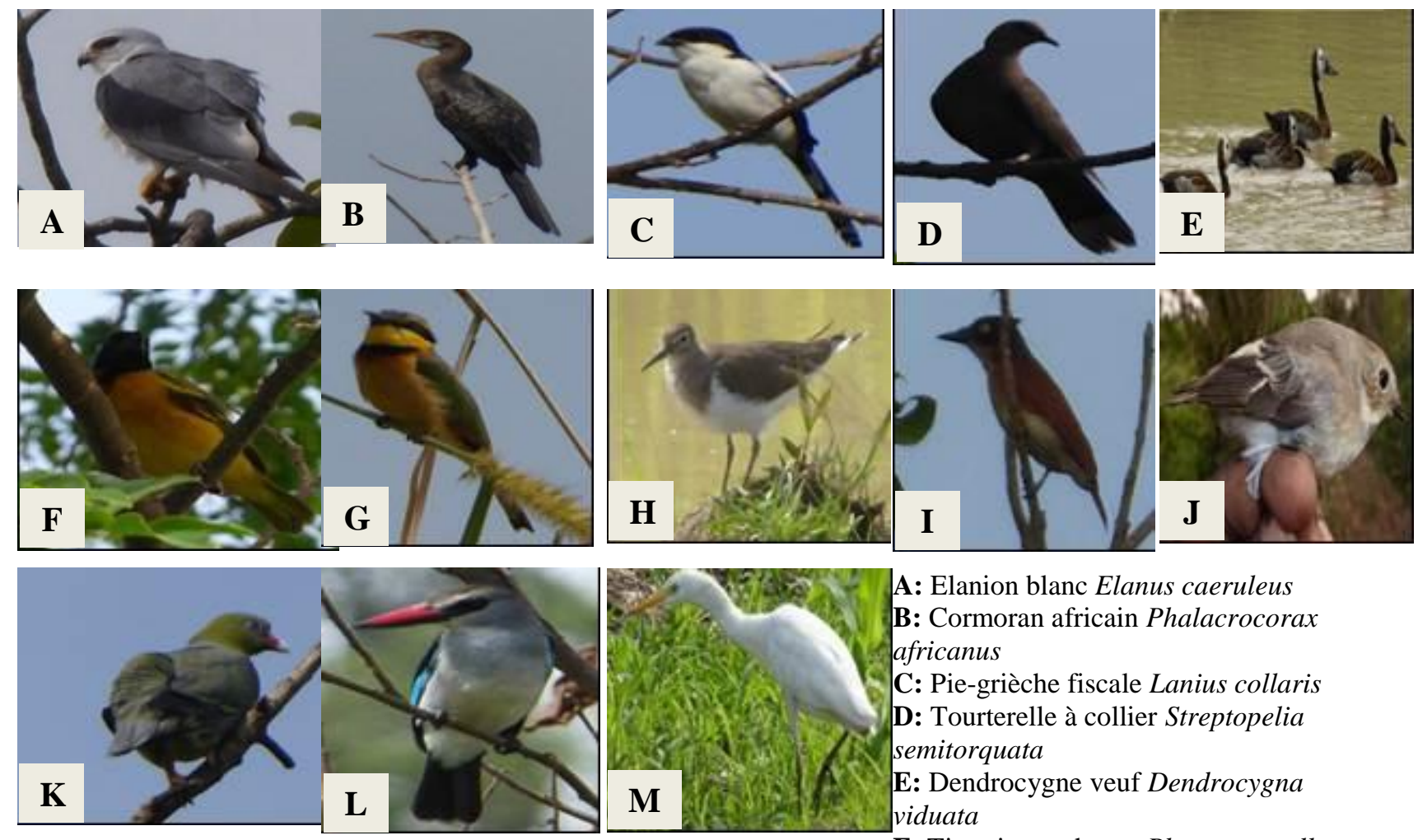

A: Elanion blanc Elanus caeruleus

B: Cormoran africain Phalacrocorax africanus

C: Pie-grièche fiscale Lanius collaris

D: Tourterelle à collier Streptopelia semitorquata

E: Dendrocygne veuf Dendrocygna viduata

F: Tisserin gendarme Ploceus cucullatus

G: Guêpier nain Merops pusillus

H: Chevalier guignette Actitis hypoleucos

I : Bias musicien Bias musicus

J : Fauvette des jardins Sylvia borin

K: Colombar à front nu Treron calvus

L: Martin-chasseur du Sénégal Halcyon senegalensis

M: Aigrette intermédiaire Egretta intermedia

Figure 3 : Photographies de quelques espèces d'oiseaux rencontrées dans le campus universitaire de Daloa (Photos Ahon). 
Tableau 1 : Liste récapitulative des espèces d'oiseaux observées sur l'ensemble des sites et leurs abondances relatives respectives.

\begin{tabular}{|c|c|c|c|c|c|c|c|c|c|c|c|c|c|}
\hline Ordres / Familles / Espèces & Noms communs & SB & $\mathbf{H P}$ & $\begin{array}{l}\text { Fr } \\
(\%)\end{array}$ & IA & SC & Bio & $\begin{array}{c}\text { Site } \\
1\end{array}$ & $\begin{array}{c}\text { Site } \\
2\end{array}$ & Site 3 & $\begin{array}{c}\text { Site } \\
4\end{array}$ & $\begin{array}{c}\text { Site } \\
5\end{array}$ & EC \\
\hline \multicolumn{14}{|l|}{ SULIFORMES } \\
\hline \multicolumn{14}{|l|}{ PHALACROCORACIDAE } \\
\hline Micrcarbo africanus (Gmelin, 1789) & Cormoran africain & $\mathrm{R}$ & $\mathrm{Ea}$ & 0,04 & Ac & $\mathrm{LC}$ & & 0 & 0 & 0 & 0 & 1 & 1 \\
\hline \multicolumn{14}{|l|}{ PELICANIFORMES } \\
\hline \multicolumn{14}{|l|}{ ARDEIDAE } \\
\hline Ardeola ralloides (Scopoli, 1769) & Crabier chevelu & $\mathrm{R} / \mathrm{P}$ & $\mathrm{Ea}$ & 0,04 & Ac & $\mathrm{LC}$ & & 0 & 0 & 0 & 0 & 1 & 1 \\
\hline Bubulcus ibis (Linné, 1758) & Héron garde-bœufs & $\mathrm{R} / \mathrm{M}$ & $\mathrm{Ea}$ & 0,66 & $\mathrm{Ra}$ & $\mathrm{LC}$ & & 0 & 0 & 0 & 16 & 0 & 16 \\
\hline Butorides striata (Linné, 1758) & Héron strié & $\mathrm{R}$ & $\mathrm{Ea}$ & 0,33 & $\mathrm{Ra}$ & $\mathrm{LC}$ & & 0 & 0 & 0 & 4 & 4 & 8 \\
\hline Egretta garzetta (Linné, 1766) & Aigrette garzette & $\mathrm{R} / \mathrm{M} / \mathrm{P}$ & $\mathrm{Ea}$ & 0,41 & $\mathrm{Ra}$ & $\mathrm{LC}$ & & 6 & 0 & 0 & 3 & 1 & 10 \\
\hline Egretta intermedia (Wagler, 1829) & Aigrette intermédiaire & $\mathrm{R} / \mathrm{M}$ & $\mathrm{Ea}$ & 7,54 & $\mathrm{D}$ & $\mathrm{LC}$ & & 0 & 48 & 32 & 72 & 30 & 182 \\
\hline Egretta alba (Linné, 1758) & Grande Aigrette & $\mathrm{R} / \mathrm{M} / \mathrm{P}$ & $\mathrm{Ea}$ & 0,08 & Ac & $\mathrm{LC}$ & & 0 & 0 & 0 & 2 & 0 & 2 \\
\hline \multicolumn{14}{|l|}{ ANSERIFORMES } \\
\hline \multicolumn{14}{|l|}{ ANATIDAE } \\
\hline Dendrocygna viduata (Linné, 1766) & Dendrocygne veuf & $\mathrm{R} / \mathrm{M}$ & $\mathrm{Ea}$ & 3,15 & $\mathrm{Re}$ & $\mathrm{LC}$ & & 0 & 0 & 0 & 44 & 32 & 76 \\
\hline \multicolumn{14}{|l|}{ ACCIPITRIFORMES } \\
\hline \multicolumn{14}{|l|}{ ACCIPITRIDAE } \\
\hline $\begin{array}{l}\text { Elanus caeruleus (Desfontaines, } \\
1789 \text { ) }\end{array}$ & Elanion blanc & $\mathrm{R}$ & $\mathrm{f}$ & 0,08 & Ac & $\mathrm{LC}$ & & 0 & 0 & 0 & 1 & 1 & 2 \\
\hline Circus macrourus (Gmelin, 1770) & Busard pâle & $\mathrm{P}$ & $\mathrm{f}$ & 0,12 & Ac & NT & & 3 & 0 & 0 & 0 & 0 & 3 \\
\hline Milvus migrans (Boddaert, 1783) & Milan noir & $\mathrm{M} / \mathrm{P} / \mathrm{R}$ & $\mathrm{f}$ & 0,62 & $\mathrm{Ra}$ & $\mathrm{LC}$ & & 2 & 4 & 4 & 3 & 2 & 15 \\
\hline Accipiter badius (Gmelin, 1788) & Epervier shikra & $\mathrm{R} / \mathrm{M}$ & $\mathrm{f}$ & 0,29 & $\mathrm{Ra}$ & $\mathrm{LC}$ & & 1 & 0 & 3 & 1 & 2 & 7 \\
\hline $\begin{array}{l}\text { Kaupifalco monogrammicus } \\
\text { (Temminck, 1824) }\end{array}$ & Autour unibande & $\mathrm{R}$ & $\mathrm{f}$ & 0,41 & $\mathrm{Ra}$ & $\mathrm{LC}$ & & 3 & 2 & 1 & 1 & 3 & 10 \\
\hline Buteo auguralis (Salvadori, 1865) & Buse d'Afrique & $\mathrm{R} / \mathrm{M}$ & $\mathrm{f}$ & 0,12 & Ac & $\mathrm{LC}$ & & 2 & 1 & 0 & 0 & 0 & 3 \\
\hline \multicolumn{14}{|l|}{ FALCONIFORMES } \\
\hline \multicolumn{14}{|l|}{ FALCONIDAE } \\
\hline Falco tinnunculus (Linné, 1758) & Faucon crécerelle & $\mathrm{R} / \mathrm{P}$ & $\mathrm{f}$ & 0,12 & Ac & $\mathrm{LC}$ & & 0 & 0 & 3 & 0 & 0 & 3 \\
\hline Falco ardosiaceus (Vieillot, 1823) & Faucon ardoisé & $\mathrm{R}$ & $\mathrm{f}$ & 0,08 & Ac & $\mathrm{LC}$ & & 0 & 1 & 0 & 0 & 1 & 2 \\
\hline Falco biarmicus (Temminck, 1825) & Faucon lanier & M & $\mathrm{f}$ & 0,04 & Ac & $\mathrm{LC}$ & & 0 & 1 & 0 & 0 & 0 & 1 \\
\hline
\end{tabular}


G. M. ZEAN et al. / Int. J. Biol. Chem. Sci. 12(6): 2503-2518, 2018

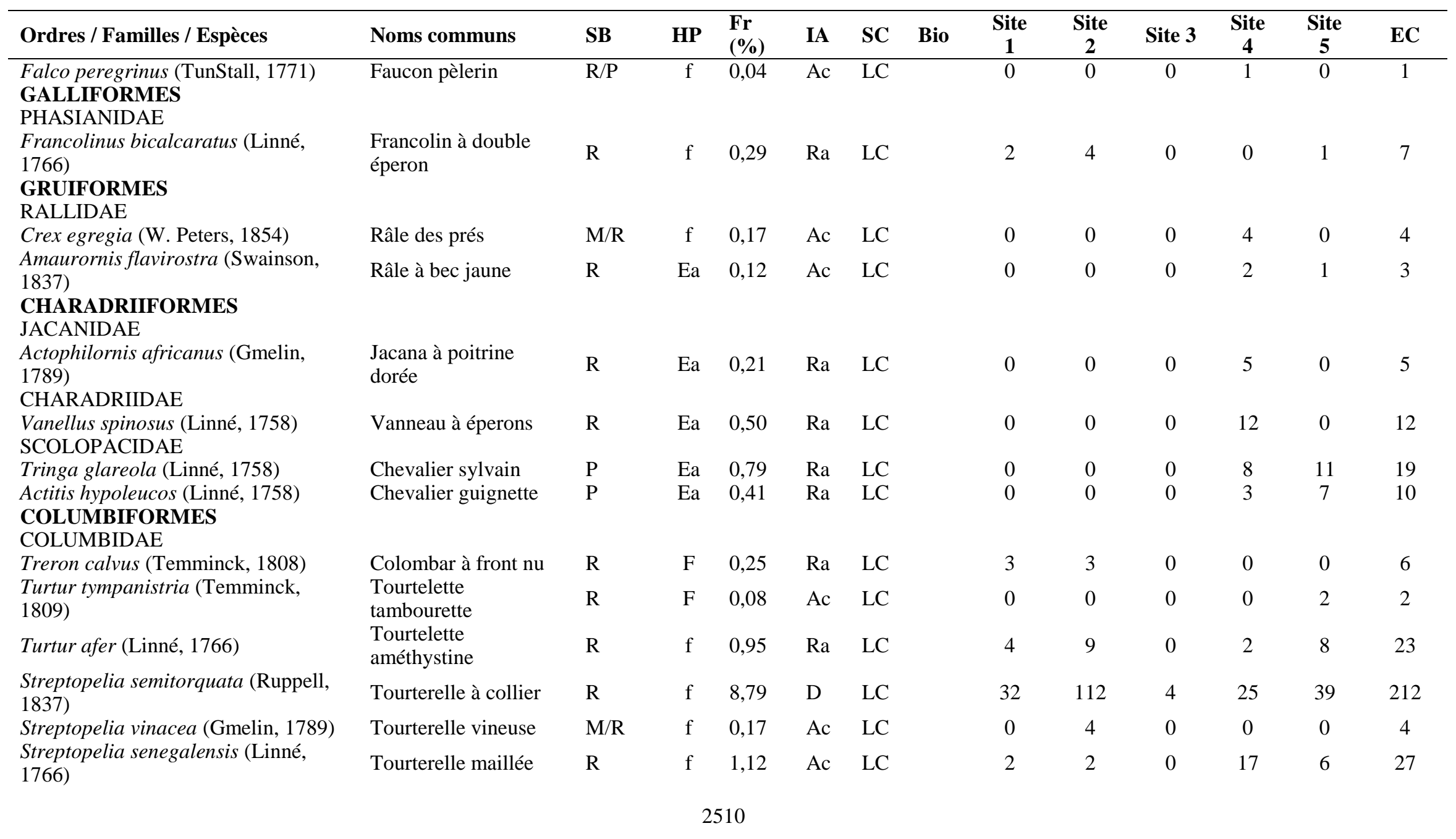


G. M. ZEAN et al. / Int. J. Biol. Chem. Sci. 12(6): 2503-2518, 2018

\begin{tabular}{|c|c|c|c|c|c|c|c|c|c|c|c|c|c|}
\hline Ordres / Familles / Espèces & Noms communs & SB & HP & $\begin{array}{l}\mathbf{F r} \\
(\%)\end{array}$ & IA & SC & Bio & $\begin{array}{c}\text { Site } \\
1\end{array}$ & $\begin{array}{c}\text { Site } \\
2\end{array}$ & Site 3 & $\begin{array}{c}\text { Site } \\
4\end{array}$ & $\begin{array}{c}\text { Site } \\
5\end{array}$ & EC \\
\hline $\begin{array}{l}\text { MUSOPHAGIFORMES } \\
\text { MUSOPHAGIDAE }\end{array}$ & & & & & & & & & & & & & \\
\hline $\begin{array}{l}\text { Crinifer piscator (Boddaert, 1783) } \\
\text { CUCULIFORMES } \\
\text { CUCULIDAE }\end{array}$ & Crinifer piscator & $\mathrm{R}$ & $\mathrm{f}$ & 0,62 & $\mathrm{Ra}$ & $\mathrm{LC}$ & & 2 & 0 & 0 & 4 & 9 & 15 \\
\hline $\begin{array}{l}\text { Oxylophus levaillantii (Swainson, } \\
\text { 1829) }\end{array}$ & Coucou de Levaillant & M & $\mathrm{Ea}$ & 0,04 & Ac & $\mathrm{LC}$ & & 0 & 1 & 0 & 0 & 0 & 1 \\
\hline $\begin{array}{l}\text { Pachycoccyx audeberti (Schlegel, } \\
\text { 1879) }\end{array}$ & Coucou d'Audebert & $\mathrm{R}$ & $\mathrm{F}$ & 0,04 & Ac & $\mathrm{LC}$ & & 1 & 0 & 0 & 0 & 0 & 1 \\
\hline Chrysococcyx klaas (Stephens, 1815) & Coucou de Klaas & $\mathrm{M} / \mathrm{R}$ & $\mathrm{f}$ & 0,12 & Ac & $\mathrm{LC}$ & & 1 & 1 & 0 & 0 & 1 & 3 \\
\hline $\begin{array}{l}\text { Chrysococcyx caprius (Boddaert, } \\
1783 \text { ) }\end{array}$ & Coucou didric & $\mathrm{M} / \mathrm{R}$ & $\mathrm{f}$ & 0,12 & Ac & $\mathrm{LC}$ & & 3 & 0 & 0 & 0 & 0 & 3 \\
\hline $\begin{array}{l}\text { Ceuthmochares aereus (Vieillot, } \\
\text { 1817) }\end{array}$ & Malcoha à bec jaune & $\mathrm{R}$ & $\mathrm{F}$ & 0,04 & Ac & $\mathrm{LC}$ & & 0 & 0 & 0 & 0 & 1 & 1 \\
\hline $\begin{array}{l}\text { Centropus senegalensis (Linné, 1766) } \\
\text { STRIGIFORMES } \\
\text { TYTONIDAE }\end{array}$ & Coucal de Sénégal & $\mathrm{R}$ & $\mathrm{f}$ & 1,12 & $\mathrm{Re}$ & $\mathrm{LC}$ & & 7 & 2 & 7 & 6 & 5 & 27 \\
\hline $\begin{array}{l}\text { Tyto alba (Scopoli, 1749) } \\
\text { STRIGIDAE }\end{array}$ & Effraie des clochers & $\mathrm{R}$ & $\mathrm{f}$ & 0,25 & $\mathrm{Ra}$ & $\mathrm{LC}$ & & 0 & 3 & 1 & 2 & 0 & 6 \\
\hline Ptilopsis leucotis (Temminck, 1820) & $\begin{array}{l}\text { Petit-duc à face } \\
\text { blanche }\end{array}$ & $\mathrm{R}$ & $\mathrm{f}$ & 0,21 & $\mathrm{Ra}$ & $\mathrm{LC}$ & & 2 & 0 & 1 & 1 & 1 & 5 \\
\hline $\begin{array}{l}\text { CAPRIMULGIFORMES } \\
\text { CAPRIMULGIDAE } \\
\text { Caprimulgus europaeus } \\
\text { CORACIIFORMES } \\
\text { ALCEDINIDAE }\end{array}$ & Engoulevent d'Europe & $\mathrm{P}$ & $\mathrm{f}$ & 0,04 & Ac & $\mathrm{LC}$ & & 0 & 0 & 0 & 1 & 0 & 1 \\
\hline Halcyon malimbica (Shaw, 1811) & $\begin{array}{l}\text { Martin-chasseur à } \\
\text { poitrine bleue }\end{array}$ & $\mathrm{R}$ & $\mathrm{F}$ & 0,041 & $\mathrm{Ac}$ & $\mathrm{LC}$ & & 1 & 0 & 0 & 0 & 0 & 1 \\
\hline Halcyon senegalensis (Linné, 1766) & $\begin{array}{l}\text { Martin-chasseur du } \\
\text { Sénégal }\end{array}$ & $\mathrm{R}$ & $\mathrm{f}$ & 0,46 & $\mathrm{Ra}$ & $\mathrm{LC}$ & & 4 & 1 & 0 & 1 & 5 & 11 \\
\hline
\end{tabular}


G. M. ZEAN et al. /Int. J. Biol. Chem. Sci. 12(6): 2503-2518, 2018

\begin{tabular}{|c|c|c|c|c|c|c|c|c|c|c|c|c|c|}
\hline Ordres / Familles / Espèces & Noms communs & SB & HP & $\begin{array}{l}\mathbf{F r} \\
(\%)\end{array}$ & IA & SC & Bio & $\begin{array}{c}\text { Site } \\
1 \\
\end{array}$ & $\begin{array}{c}\text { Site } \\
2 \\
\end{array}$ & Site 3 & $\begin{array}{c}\text { Site } \\
4 \\
\end{array}$ & $\begin{array}{c}\text { Site } \\
5\end{array}$ & EC \\
\hline Ceyx lecontei (Schlegel, 1879) & $\begin{array}{l}\text { Martin-pêcheur à tête } \\
\text { rousse }\end{array}$ & $\mathrm{R}$ & f & 0,17 & Ac & $\mathrm{LC}$ & A05 & 0 & 0 & 0 & 0 & 4 & 4 \\
\hline Ceyx pictus (Boddaert, 1783) & $\begin{array}{l}\text { Martin-pêcheur } \\
\text { pygmée }\end{array}$ & $\mathrm{R} / \mathrm{M}$ & $\mathrm{f}$ & 0,46 & $\mathrm{Ra}$ & $\mathrm{LC}$ & & 0 & 4 & 0 & 1 & 6 & 11 \\
\hline Alcedo leucogaster (Fraser, 1843) & $\begin{array}{l}\text { Martin-pêcheur à } \\
\text { ventre blanc }\end{array}$ & $\mathrm{R}$ & $\mathrm{f}$ & 0,08 & Ac & $\mathrm{LC}$ & A05 & 0 & 0 & 0 & 1 & 1 & 2 \\
\hline $\begin{array}{l}\text { Alcedo cristata (Pallas, 1764) } \\
\text { MEROPIDAE }\end{array}$ & Martin-pêcheur huppé & $\mathrm{R} / \mathrm{M}$ & $\mathrm{Ea}$ & 0,08 & Ac & $\mathrm{LC}$ & & 0 & 1 & 0 & 0 & 1 & 2 \\
\hline Merops pusillus (Muller, 1776) & Guêpier nain & $\mathrm{R}$ & $\mathrm{f}$ & 0,17 & Ac & $\mathrm{LC}$ & & 0 & 0 & 1 & 1 & 2 & 4 \\
\hline Merops albicollis (Vieillot, 1817) & $\begin{array}{l}\text { Guêpier à gorge } \\
\text { blanche }\end{array}$ & M & $\mathrm{f}$ & 8 & $\mathrm{D}$ & $\mathrm{LC}$ & & 117 & 20 & 0 & 56 & 0 & 193 \\
\hline CORACIIDAE & & & & & & & & & & & & & \\
\hline Coracias cyanogaster (Cuvier, 1816) & Rollier à ventre bleu & $\mathrm{R} / \mathrm{M}$ & $\mathrm{f}$ & 0,04 & Ac & $\mathrm{LC}$ & & 0 & 0 & 0 & 0 & 1 & 1 \\
\hline $\begin{array}{l}\text { Coracias abyssinicus (Hermann, } \\
1783 \text { ) }\end{array}$ & Rollier d'Abyssinie & M & $\mathrm{F}$ & 0,12 & Ac & $\mathrm{LC}$ & A04 & 2 & 1 & 0 & 0 & 0 & 3 \\
\hline Eurystomus gularis (Vieillot, 1819) & Rolle à gorge bleue & $\mathrm{R} / \mathrm{M}$ & $\mathrm{F} / \mathrm{f}$ & 0,08 & Ac & $\mathrm{LC}$ & A05 & 0 & 1 & 0 & 0 & 1 & 2 \\
\hline $\begin{array}{l}\text { Eurystomus glaucurus (Statius } \\
\text { Muller, 1776) } \\
\text { BUCEROTIFORMES } \\
\text { BUCEROTIDAE }\end{array}$ & Rolle violet & M & $\mathrm{f}$ & 0,33 & $\mathrm{Ra}$ & $\mathrm{LC}$ & & 0 & 1 & 1 & 2 & 4 & 8 \\
\hline Tockus fasciatus (Shaw, 1811) & Calao longibande & $\mathrm{R}$ & $\mathrm{F}$ & 0,29 & $\mathrm{Ra}$ & $\mathrm{LC}$ & A05 & 2 & 2 & 0 & 1 & 2 & 7 \\
\hline $\begin{array}{l}\text { Tockus nasutus (Linné, 1766) } \\
\text { PICIFORMES } \\
\text { LYBIIDAE }\end{array}$ & Calao à bec noir & $\mathrm{R}$ & $\mathrm{f}$ & 0,08 & Ac & $\mathrm{LC}$ & & 0 & 1 & 1 & 0 & 0 & 2 \\
\hline Lybius vieilloti (Leach, 1815) & Barbican de Vieillot & $\mathrm{R}$ & f & 0,66 & $\mathrm{Ra}$ & $\mathrm{LC}$ & & 4 & 2 & 0 & 4 & 6 & 16 \\
\hline $\begin{array}{l}\text { Lybius bidentatus (Shaw, 1799) } \\
\text { PICIDAE }\end{array}$ & Barbican bidenté & $\mathrm{R}$ & $\mathrm{f}$ & 0,04 & Ac & $\mathrm{LC}$ & & 0 & 0 & 0 & 0 & 1 & 1 \\
\hline $\begin{array}{l}\text { Dendropicos goertae (Statius Muller, } \\
\text { 1776) } \\
\text { PASSERIFORMES } \\
\text { HIRUNDINIDAE }\end{array}$ & Pic goertan & $\mathrm{R}$ & $\mathrm{F}$ & 0,25 & $\mathrm{Ra}$ & $\mathrm{LC}$ & & 6 & 0 & 0 & 0 & 0 & 6 \\
\hline
\end{tabular}


G. M. ZEAN et al. /Int. J. Biol. Chem. Sci. 12(6): 2503-2518, 2018

\begin{tabular}{|c|c|c|c|c|c|c|c|c|c|c|c|c|c|}
\hline Ordres / Familles / Espèces & Noms communs & SB & HP & $\begin{array}{l}\mathbf{F r} \\
(\%)\end{array}$ & IA & SC & Bio & $\begin{array}{c}\text { Site } \\
1\end{array}$ & $\begin{array}{c}\text { Site } \\
2\end{array}$ & Site 3 & $\begin{array}{c}\text { Site } \\
4\end{array}$ & $\begin{array}{c}\text { Site } \\
5\end{array}$ & EC \\
\hline Psalidoprocne nitens (Cassin, 1857) & $\begin{array}{l}\text { Hirondelle à queue } \\
\text { courte }\end{array}$ & $\mathrm{R}$ & $\mathrm{F}$ & 0,50 & $\mathrm{Ra}$ & $\mathrm{LC}$ & A05 & 0 & 0 & 2 & 10 & 0 & 12 \\
\hline $\begin{array}{l}\text { Psalidoprocne obscura (Hartlaub, } \\
\text { 1855) }\end{array}$ & Hirondelle fanti & $\mathrm{R} / \mathrm{M}$ & $\mathrm{F}$ & 0,25 & $\mathrm{Ra}$ & $\mathrm{LC}$ & A05 & 0 & 0 & 0 & 0 & 6 & 6 \\
\hline Hirundo aethiopica (Blanford, 1869) & Hirondelle d'Ethiopie & $\mathrm{R} / \mathrm{M}$ & $\mathrm{f}$ & 0,25 & $\mathrm{Ra}$ & $\mathrm{LC}$ & & 0 & 0 & 0 & 6 & 0 & 6 \\
\hline $\begin{array}{l}\text { Hirundo abyssinica (Guerin- } \\
\text { Meneville, 1843) }\end{array}$ & Hirondelle striée & $\mathrm{R} / \mathrm{M}$ & $\mathrm{f}$ & 2,32 & $\operatorname{Re}$ & $\mathrm{LC}$ & & 0 & 0 & 0 & 16 & 40 & 56 \\
\hline Hirundo preussi (Reichenow, 1898) & Hirondelle de Preuss & $\mathrm{R}$ & $\mathrm{f}$ & 0,50 & $\mathrm{Ra}$ & $\mathrm{LC}$ & & 0 & 0 & 0 & 12 & 0 & 12 \\
\hline $\begin{array}{l}\text { Delichon urbicum (Linné, 1758) } \\
\text { MOTACILLIDAE }\end{array}$ & Hirondelle de fenêtre & $\mathrm{M} / \mathrm{P}$ & & 1,16 & Ac & $\mathrm{LC}$ & & 16 & 2 & 0 & 10 & 0 & 28 \\
\hline Motacilla flava (Linné, 1758) & $\begin{array}{l}\text { Bergeronnette } \\
\text { printanière }\end{array}$ & $\mathrm{P}$ & $\mathrm{f}$ & 0,21 & $\mathrm{Ra}$ & $\mathrm{LC}$ & & 0 & 0 & 0 & 3 & 2 & 5 \\
\hline Anthus leucophrys (Vieillot, 1818) & Pipit à dos uni & $\mathrm{R}$ & $\mathrm{f}$ & 0,58 & $\mathrm{Ra}$ & $\mathrm{LC}$ & & 0 & 0 & 0 & 14 & 0 & 14 \\
\hline Anthus cervinus (Pallas, 1811) & Pipit à gorge rousse & $\mathrm{P}$ & $\mathrm{f}$ & 0,04 & Ac & $\mathrm{LC}$ & & 0 & 0 & 0 & 1 & 0 & 1 \\
\hline Macronyx croceus (Vieillot, 1816) & $\begin{array}{l}\text { Sentinelle à gorge } \\
\text { jaune }\end{array}$ & $\mathrm{R}$ & $\mathrm{f}$ & 0,41 & $\mathrm{Ra}$ & $\mathrm{LC}$ & & 0 & 0 & 0 & 10 & 0 & 10 \\
\hline $\begin{array}{l}\text { PYCNONOTIDAE } \\
\text { Eurylas virens Cassin, } 1858\end{array}$ & Bulbul verdâtre & $\mathrm{R}$ & $\mathrm{f}$ & 1,66 & $\operatorname{Re}$ & $\mathrm{LC}$ & & 15 & 16 & 0 & 0 & 9 & 40 \\
\hline $\begin{array}{l}\text { Pycnonotus barbatus (Desfontaines, } \\
\text { 1789) } \\
\text { TURDIDAE }\end{array}$ & Bulbul des jardins & $\mathrm{R}$ & $\mathrm{f}$ & 1,95 & $\operatorname{Re}$ & $\mathrm{LC}$ & & 5 & 4 & 13 & 21 & 4 & 47 \\
\hline $\begin{array}{l}\text { Turdus pelios Bonaparte, } 1850 \\
\text { ACCROCEPHALIDAE }\end{array}$ & Turdus pelios & $\mathrm{R}$ & $\mathrm{f}$ & 0,04 & Ac & $\mathrm{LC}$ & & 1 & 0 & 0 & 0 & 0 & 1 \\
\hline $\begin{array}{l}\text { Acrocephalus scirpaceus (Hermann, } \\
\text { 1804) }\end{array}$ & Rousserolle effarvatte & $\mathrm{P}$ & $\mathrm{f}$ & 0,04 & Ac & $\mathrm{LC}$ & & 1 & 0 & 0 & 0 & 0 & 1 \\
\hline $\begin{array}{l}\text { Acrocephalus arundinaceus (Linné, } \\
\text { 1758) } \\
\text { CISTICOLIDAE }\end{array}$ & Rousserolle turdoïde & $\mathrm{P}$ & $\mathrm{Ea}$ & 0,17 & Ac & $\mathrm{LC}$ & & 0 & 0 & 0 & 0 & 4 & 4 \\
\hline Cisticola erythrops (Hartlaub, 1857) & $\begin{array}{l}\text { Cisticole à face } \\
\text { rousse }\end{array}$ & $\mathrm{R}$ & $\mathrm{f}$ & 0,83 & $\mathrm{Ra}$ & $\mathrm{LC}$ & & 6 & 7 & 0 & 3 & 4 & 20 \\
\hline Cisticola lateralis (Fraser, 1843) & Cisticole siffleuse & $\mathrm{R}$ & $\mathrm{f}$ & 0,58 & $\mathrm{Ra}$ & $\mathrm{LC}$ & & 2 & 9 & 0 & 0 & 3 & 14 \\
\hline
\end{tabular}


G. M. ZEAN et al. /Int. J. Biol. Chem. Sci. 12(6): 2503-2518, 2018

\begin{tabular}{|c|c|c|c|c|c|c|c|c|c|c|c|c|c|}
\hline Ordres / Familles / Espèces & Noms communs & SB & HP & $\begin{array}{l}\mathbf{F r} \\
(\%)\end{array}$ & IA & SC & Bio & $\begin{array}{c}\text { Site } \\
1\end{array}$ & $\begin{array}{c}\text { Site } \\
2\end{array}$ & Site 3 & $\begin{array}{c}\text { Site } \\
4\end{array}$ & $\begin{array}{c}\text { Site } \\
5\end{array}$ & EC \\
\hline $\begin{array}{l}\text { Cisticola galactotes (Temminck, } \\
1821 \text { ) }\end{array}$ & Cisticole roussâtre & $\mathrm{R}$ & $\mathrm{f}$ & 0,12 & Ac & $\mathrm{LC}$ & & 1 & 1 & 0 & 0 & 1 & 3 \\
\hline $\begin{array}{l}\text { Cisticola brachypterus (Sharpe, } \\
1870 \text { ) }\end{array}$ & $\begin{array}{l}\text { Cisticole à ailes } \\
\text { courtes }\end{array}$ & $\mathrm{R}$ & $\mathrm{f}$ & 0,08 & Ac & $\mathrm{LC}$ & & 0 & 2 & 0 & 0 & 0 & 2 \\
\hline Prinia subflava (Gmelin, 1789) & Prinia modeste & $\mathrm{R}$ & $\mathrm{f}$ & 0,25 & $\mathrm{Ra}$ & $\mathrm{LC}$ & & 1 & 3 & 0 & 0 & 2 & 6 \\
\hline $\begin{array}{l}\text { Camaroptera brachyura (Vieillot, } \\
\text { 1820) }\end{array}$ & $\begin{array}{l}\text { Camaroptère à tête } \\
\text { grise }\end{array}$ & $\mathrm{R}$ & $\mathrm{f}$ & 0,21 & $\mathrm{Ra}$ & $\mathrm{LC}$ & & 0 & 4 & 0 & 0 & 1 & 5 \\
\hline $\begin{array}{l}\text { SCOTOCERIDAE } \\
\text { Hylia prasina (Cassin, 1855) } \\
\text { SYLVIIDAE }\end{array}$ & Hylia verte & $\mathrm{R}$ & $\mathrm{F}$ & 0,17 & $\mathrm{Ac}$ & $\mathrm{LC}$ & A05 & 1 & 3 & 0 & 0 & 0 & 4 \\
\hline Sylvietta virens (Cassin, 1859) & Crombec vert & $\mathrm{R}$ & $\mathrm{F}$ & 0,17 & Ac & $\mathrm{LC}$ & A05 & 1 & 2 & 0 & 0 & 1 & 4 \\
\hline $\begin{array}{l}\text { Sylvia borin (Boddaert, 1783) } \\
\text { MUSCICAPIDAE }\end{array}$ & Fauvette des jardins & $\mathrm{P}$ & $\mathrm{f}$ & 0,04 & Ac & $\mathrm{LC}$ & & 0 & 1 & 0 & 0 & 0 & 1 \\
\hline Saxicola rubetra (Linné, 1758) & Tarier des prés & $\mathrm{P}$ & f & 0,46 & $\mathrm{Ra}$ & $\mathrm{LC}$ & & 0 & 0 & 0 & 7 & 4 & 11 \\
\hline $\begin{array}{l}\text { Melaenornis edolioides (Swainson, } \\
\text { 1837) } \\
\text { PLATYSTEIRIDAE }\end{array}$ & Gobemouche drongo & $\mathrm{R}$ & $\mathrm{f}$ & 0,04 & Ac & $\mathrm{LC}$ & & 1 & 0 & 0 & 0 & 0 & 1 \\
\hline $\begin{array}{l}\text { Bias musicus (Vieillot, 1818) } \\
\text { PARIDAE }\end{array}$ & Bias musicien & $\mathrm{R}$ & $\mathrm{f}$ & 0,25 & $\mathrm{Ra}$ & $\mathrm{LC}$ & & 1 & 1 & 0 & 0 & 4 & 6 \\
\hline $\begin{array}{l}\text { Melaniparus guineensis Shelley, } \\
1900 \\
\text { NECTARINIIDAE }\end{array}$ & Mésange galonnée & $\mathrm{R}$ & $\mathrm{f}$ & 0,04 & Ac & $\mathrm{LC}$ & & 0 & 1 & 0 & 0 & 0 & 1 \\
\hline Hedydipna collaris (Vieillot, 1819) & Souimanga à collier & $\mathrm{R}$ & $\mathrm{F}$ & 0,12 & Ac & $\mathrm{LC}$ & & 0 & 0 & 0 & 0 & 3 & 3 \\
\hline Cinnyris chloropygius (Jardine, 1842) & $\begin{array}{l}\text { Souimanga à ventre } \\
\text { olive }\end{array}$ & $\mathrm{R}$ & $\mathrm{F} / \mathrm{f}$ & 0,04 & $\mathrm{Ac}$ & $\mathrm{LC}$ & & 1 & 0 & 0 & 0 & 0 & 1 \\
\hline $\begin{array}{l}\text { Cinnyris cupreus (Shaw, 1812) } \\
\text { LANIIDAE }\end{array}$ & Souimanga cuivré & $\mathrm{R}$ & $\mathrm{f}$ & 0,58 & $\mathrm{Ra}$ & $\mathrm{LC}$ & & 4 & 4 & 0 & 1 & 5 & 14 \\
\hline $\begin{array}{l}\text { Lanius collaris (Linné, 1766) } \\
\text { MALACONOTIDAE }\end{array}$ & Pie-grièche fiscale & $\mathrm{R}$ & $\mathrm{f}$ & 0,87 & $\mathrm{Ra}$ & $\mathrm{LC}$ & & 5 & 2 & 0 & 10 & 4 & 21 \\
\hline $\begin{array}{l}\text { Tchagra senegalus (Linné, 1766) } \\
\text { DICRURIDAE }\end{array}$ & Tchagra à tête noire & $\mathrm{R}$ & f & 0,50 & $\mathrm{Ra}$ & $\mathrm{LC}$ & & 3 & 3 & 0 & 3 & 3 & 12 \\
\hline
\end{tabular}


G. M. ZEAN et al. / Int. J. Biol. Chem. Sci. 12(6): 2503-2518, 2018

\begin{tabular}{|c|c|c|c|c|c|c|c|c|c|c|c|c|c|}
\hline Ordres / Familles / Espèces & Noms communs & SB & HP & $\begin{array}{l}\text { Fr } \\
(\%)\end{array}$ & IA & SC & Bio & $\begin{array}{c}\text { Site } \\
1\end{array}$ & $\begin{array}{c}\text { Site } \\
2\end{array}$ & Site 3 & $\begin{array}{c}\text { Site } \\
4\end{array}$ & $\begin{array}{c}\text { Site } \\
5\end{array}$ & EC \\
\hline $\begin{array}{l}\text { Dicrurus modestus Hartlaub, } 1849 \\
\text { CORVIDAE }\end{array}$ & Drongo modeste & $\mathrm{R}$ & $\mathrm{F}$ & 0,25 & $\mathrm{Ra}$ & $\mathrm{LC}$ & & 3 & 0 & 0 & 0 & 3 & 6 \\
\hline $\begin{array}{l}\text { Corvus albus Müller, } 1776 \\
\text { STURNIDAE }\end{array}$ & Corbeau pie & $\mathrm{R}$ & f & 2,61 & $\operatorname{Re}$ & $\mathrm{LC}$ & & 6 & 2 & 21 & 32 & 2 & 63 \\
\hline $\begin{array}{l}\text { Lamprotornis splendidus (Vieillot, } \\
\text { 1822) }\end{array}$ & Choucador splendide & $\mathrm{R}$ & $\mathrm{F}$ & 0,70 & $\mathrm{Ra}$ & $\mathrm{LC}$ & & 2 & 1 & 6 & 3 & 5 & 17 \\
\hline $\begin{array}{l}\text { PASSERIDAE } \\
\text { Passer griseus (Vieillot, 1817) } \\
\text { PLOCEIDAE }\end{array}$ & Moineau gris & $\mathrm{R}$ & f & 7,21 & $\mathrm{D}$ & $\mathrm{LC}$ & & 0 & 0 & 15 & 129 & 30 & 174 \\
\hline Ploceus pelzelni (Hartlaub, 1887) & Tisserin de Pelzeln & $\mathrm{R}$ & $f$ & 0,04 & Ac & $\mathrm{LC}$ & & 0 & 1 & 0 & 0 & 0 & 1 \\
\hline Ploceus nigerrimus Vieillot, 1819 & Tisserin noir & $\mathrm{R}$ & f & 0,58 & $\mathrm{Ra}$ & LC & A05 & 2 & 0 & 0 & 0 & 12 & 14 \\
\hline Ploceus cucullatus (Müller, 1776) & Tisserin gendarme & $\mathrm{R}$ & $f$ & 10,15 & $\mathrm{D}$ & $\mathrm{LC}$ & & 44 & 52 & 0 & 147 & 2 & 245 \\
\hline $\begin{array}{l}\text { Euplectes afer (J. F. Gmelin, 1789) } \\
\text { ESTRILDIDAE }\end{array}$ & Euplecte vorabé & $\mathrm{R}$ & f & 0,50 & $\mathrm{Ra}$ & $\mathrm{LC}$ & & 12 & 0 & 0 & 0 & 0 & 12 \\
\hline Estrilda melpoda (Vieillot, 1817) & Astrild à joues orange & $\mathrm{R}$ & $f$ & 0,17 & Ac & $\mathrm{LC}$ & & 0 & 1 & 0 & 3 & 0 & 4 \\
\hline Lagonosticta senegala (Linné, 1766) & Amarante du Sénégal & $\mathrm{R}$ & f & 0,21 & $\mathrm{Ra}$ & $\mathrm{LC}$ & & 0 & 0 & 0 & 0 & 5 & 5 \\
\hline $\begin{array}{l}\text { Spermestes cucullatus (Swainson, } \\
1837 \text { ) }\end{array}$ & Capucin nonnette & $\mathrm{R}$ & $\mathrm{f}$ & 18,20 & $\mathrm{D}$ & $\mathrm{LC}$ & & 49 & 47 & 21 & 299 & 23 & 439 \\
\hline $\begin{array}{l}\text { Spermestes bicolor (Fraser, 1843) } \\
\text { VIDUIDAE }\end{array}$ & Capucin bicolore & $\mathrm{R}$ & $\mathrm{f}$ & 1,04 & $\operatorname{Re}$ & $\mathrm{LC}$ & & 17 & 2 & 0 & 0 & 6 & 25 \\
\hline Vidua chalybeata (Müller, 1776) & $\begin{array}{l}\text { Combassou du } \\
\text { Sénégal }\end{array}$ & $\mathrm{R}$ & $\mathrm{f}$ & 0,75 & $\mathrm{Ra}$ & $\mathrm{LC}$ & & 0 & 0 & 0 & 0 & 18 & 18 \\
\hline Vidua macroura (Pallas, 1764) & Veuve dominicaine & $\mathrm{R}$ & $f$ & 0,17 & Ac & $\mathrm{LC}$ & & 0 & 0 & 0 & 1 & 3 & 4 \\
\hline Totaux & & & & 100 & & & & 412 & 403 & 137 & 1048 & 413 & 2413 \\
\hline Richesse spécifiques (Rs) & & & & & & & & 49 & 50 & 18 & 55 & 65 & 105 \\
\hline Indice de Shannon (H') & & & & & & & & 2,82 & 2,70 & 2,32 & 2,70 & 3,52 & \\
\hline Indice d'Equitabilité (E) & $\mathrm{CH}_{\mathrm{C}}+\mathrm{CH}+\mathrm{CH}+\mathrm{CH}$ & & & 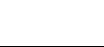 & & & & 0,72 & 0,69 & 0,80 & 0,67 & 0,84 & \\
\hline
\end{tabular}




\section{DISCUSSION}

La richesse spécifique obtenue au sein de la zone d'étude représente $13,85 \%$ des espèces d'oiseaux rencontrées dans le pays qui est de 758 espèces (Ahon, 2016). Quoique relativement faible, cette richesse spécifique d'oiseaux apparaît diversifiée eu égard à la courte période d'étude. Celle-ci pourrait s'expliquer par la diversité des habitats et des ressources alimentaires offertes par les zones d'étude respectives. En effet, la présence de la carrière offre des points d'eau non permanents par endroit, la décharge d'ordure à ciel ouvert, la riziculture et la plantation d'anacardier attirent de nombreuses espèces d'oiseaux comme l'ont démontré les travaux de Konan et al. (2015). La présence de l'espèce Circus macrourus, dont la protection est d'intérêt mondial (UICN, 2018) montre que le CUJLoG mériterait une attention particulière. Le statut biogéographique révèle par ailleurs une proportion de $14,28 \%$ d'espèces totalement migratrices parmi les spécimens recensés. Cette proportion paraît peu significative en comparaison des 151 espèces d'oiseaux totalement migratrices susceptibles d'être rencontrées sur le territoire ivoirien (Ahon, 2016). Ces faibles données relatives aux espèces migratrices pourraient se justifier par le fait que le CUJLoG ne soit pas favorable à toutes les espèces totalement migratrices, car n'abritant certainement pas les conditions écologiques idoines pour ces espèces non observées. Toutefois, le peuplement est dominé par des espèces résidentes. Cela pourrait se traduire par le fait que ces espèces trouvent durant l'année ou la période d'étude les conditions écologiques favorables à leur vie (reproduction, alimentation et habitats préférentiels) comme l'ont signifié Kouadio et al. (2014). La prédominance des espèces des habitats ouverts serait liée à l'urbanisation ou à l'anthropisation. Et les quelques espèces forestières qui ont été observées s'expliquerait par la présence de la forêt secondaire qui constitue un refuge pour ces espèces. Cette hypothèse est soutenue par les observations de Yaokokoré-Béibro et al. (2015a) dans la commune de Cocody (Abidjan, Côte d'Ivoire). En termes de richesse spécifique, en comparaison avec les données obtenues dans d'autres sites urbains de Côte d'Ivoire, le nombre d'espèces d'oiseaux du CUJLoG est plus élevé que celui du milieu urbain de la commune de Cocody (Yaokokoré-Béibro et al., 2015a) et plus faible que celui de la zone urbaine de Grand-Bassam (Yaokokoré-Béibro et al., 2015b). Par contre, il est sensiblement égal à celui obtenu par Konan et al., 2015 dans la ville de Yamoussoukro. Ces différences et ces similitudes pourraient s'expliquer non seulement par l'étendue et la diversité des habitats des différents sites d'étude, mais également par la période choisie pour les prospections avifauniques dans les différentes études. La zone de carrière a l'indice de diversité le plus élevé certainement à cause de sa structure et de la diversité de ressources alimentaires qu'elle offrirait aux oiseaux. Par contre les zones faiblement diversifiées sont dues aux perturbations liées aux activités humaines.

\section{Conclusion}

L'objectif de l'étude était de contribuer à une meilleure connaissance de l'avifaune urbaine de la Côte d'Ivoire. Cette étude a montré que le CUJLoG et sa périphérie sont riches et très diversifiés avec 105 espèces de 43 familles et 17 ordres. Parmi les espèces inventoriées, l'espèce Spermestes cucullatus domine avec $18,19 \%$ de l'effectif total. Les espèces résidentes $(63,81 \%)$ et celles des milieux ouverts $(68,57 \%)$ sont majoritaires dans les zones d'étude. Aussi, en plus des neuf espèces d'oiseaux inféodées au biome des forêts guinéo-congolaise et d'une espèce de la savane soudano-guinéenne, une espèce Circus macrourus inscrite sur la liste rouge de l'UICN et classée quasi-menacée a-t-elle été recensée dans la zone dégradée. Cela démontre que le CUJLoG apparait être un milieu d'intérêt pour la conservation des oiseaux.

\section{CONFLIT D'INTERETS}

Les auteurs déclarent n'avoir aucun conflit d'intérêts entre eux.

\section{CONTRIBUTIONS DES AUTEURS}

GMZ a assuré la conduite des travaux sur le terrain et a contribué à la rédaction de l'article. DBA a assuré la conception du protocole, contribué à la réalisation des travaux de terrain et à la rédaction du manuscrit. BJCK a contribué à la rédaction du manuscrit et a assuré la direction scientifique de l'étude. 


\section{REMERCIEMENTS}

Les auteurs remercient toutes les personnes qui ont contribué d'une manière ou d'une autre à la réalisation de cette étude. Ce sont particulièrement la gouvernance de l'Université Jean Lorougnon Guédé et le Président de l'ONG SOS-Forêts (avec son partenaire BirdLife International) pour l'appui logistique fourni dans le cadre de cette étude.

\section{REFERENCES}

Ahon DB. 2016. Liste des oiseaux de Côte d'Ivoire. Compilation personnelle de données ornithologiques issues de rapports d'étude, non publié, $23 \mathrm{p}$.

Bamba K. 2015. Etude des produits de chasse dans le Haut Sassandra : outil d'évaluation de la faune et de la macrofaune. Master des sciences de la vie et de la terre, Université Jean Lorougnon Guédé, Daloa, Côte d'Ivoire, p. 58.

Bibby CJ, Burgess ND, Hill DA. 1992. Bird Census Techniques. Academic Press: London, England; 257 p.

BNETD (Bureau National d'Etudes Techniques et de Développement). 2007. Atlas de Daloa, Rapport d'enquête Bnetd, MOS, Daloa, Côte d'Ivoire, 12 p.

Borrow N, Demey R. 2001. Field Guide to the Birds of Western Africa. Christopher Helm: London, England, UK; 832 p.

Borrow N, Demey R. 2004. Field Guide to the Birds of Western Africa. Christopher Helm: London, UK; 511 p.

Bot JA, Nachtergaele OF, Young A. 2000. Land Resource Potentiel and Contraints at Regional and Country levels. FAO, World Soil Resources Report, Rome. Italie, $26 \mathrm{p}$.

Chace JF, Walsh JJ. 2006. Urban effects on native avifauna: a review. Landscape and Urban Planning, 74, 4-69

Chappuis C. 2000. Oiseaux d'Afrique. 15 CDs. Paris, France. Société d'étude Ornithologique.

Cincotta RPER. 2000. Nature's place: Human population and the future of biological diversity. Population Action International, Washington, D.C.

Fishpool LDC, Evans MI. 2001. Important Bird Areas in Africa and Associated Islands. BirdLife International, Cambridge, Grande Bretagne, 27p.
Gibbons DW, Gregory RD. 2006. Birds. In Ecological Census Techniques: A Handbook (2nd edn), Sutherland WJ (ed.). Cambridge University Press ; 308350.

Goudard A, 2007. Fonctionnement des écosystèmes et invasions biologiques: importance de la biodiversité et des interactions interspécifiques. Ecologie, Environnement. Thèse de Doctorat de Université Pierre et Marie Curie- Paris VI, France, p. 216.

Huillet L. 2007. Tendances spatio-temporelles de répartition chez les oiseaux hivernants durant 26 ans d'étalement urbain à Québec. Mémoire de l'Université Laval, Québec, p. 81

Konan EM, Yaokokoré-Béibro KH, Odoukpe KSG. 2015. Richesse spécifique et abondance des oiseaux des dix Lacs Urbains de la ville de Yamoussoukro, dans le centre de la Côte d'Ivoire. International Journal of Innovation and Applied Studies, 10 (1): 1-9.

Kouadio KP, Yaokokoré-Béibro KH, Odoukpe KSG, Konan EM, N'guessan AM, Kouassi KP. 2014. Diversité avifaunique de la forêt classée de N'ganda N'ganda (Sud-Est de la Côte d'Ivoire). Afrique Science, 10 (1) : 1-13.

Kouakou ATM, Barima YSS, Kouakou KA, Kouamé NF, Bogaert J, Kouadio YJ. 2015. Forest Dynamics in the North of the Classified Forest of Haut-Sassandra During the Period of Armed Conflicts in Ivory Coast. American Journal of Life Sciences, 3 (5): 375-382.

Kouakou VC, Béné J-CK, N'Guessan KA, Kouakou YC, Bamba K. 2017. Diversity, Distribution and Social Structure of Monkey Species in Forest Fragments of Gbetitapea, CentralWestern Ivory Coast. Journal of Chemical, Biological and Physical Sciences, 8 (1): 1-17.

Kouamé NF, Tra Bi HF, Etien TD, Traore D. 1998. Végétation et flore de la forêt classée du Haut-Sassandra en Côte d'Ivoire. Revue CAMES : 28-35.

Lauginie F. 2007. Conservation de la Nature et des Aires Protégés en Côte d'Ivoire. $\mathrm{NEI} / \mathrm{Hachette}$ et Afrique Nature : Abidjan ; $668 \mathrm{p}$.

Lepage D. 2018. Liste des oiseaux de Côte d'Ivoire. Avibase, la base de données 
mondiale des oiseaux. Consulté sur .https://avibase.bsc-eoc.org, le [31/10/2018].

Le Roux X, Barbault R, Baudry J, Burel F, Doussan I, Garnier E, Herzog F, Lavorel S, Lifran R, Roger-Estrade J, Sarthou JP, Trommetter M. 2008. Agriculture et biodiversité. Valoriser les synergies. Expertise scientifique collective, synthèse du rapport, INRA (France), $112 p$.

MPD : Ministère du plan et du développement. 2015. Etude monographique et économiques des districts de Côte d'Ivoire. Ministère d'Etat, Ministère du plan et du développement. Note de synthèse, $69 \mathrm{p}$.

N'Guessan KE, Kouassi RH. 2005. Accroissement en diamètre d'essences forestières au sein de trois périmètres expérimentaux (Mopri, Téné et Irobo) en Côte d'Ivoire. Agronomie Africaine, 17(2) : 91-102.

Odoukpé GSK, Yaokokoré-Béibro HK, Konan ME, Kouadio PK. 2014. L'avifaune d'un milieu de riziculture et de ses environs dans la zone humide de Grand-Bassam, sud-est Côte d'Ivoire, Malimbus, 36 (2) : 106-115.

Serles W, Morel GJ. 1993. Les Oiseaux de l'Ouest Africain. Delachaux et Niestlé : Paris, France ; 331 p.

Stattersfield AJ, Crosby MJ, Long AJ, Wege DC. 1998. Endemic Bird Areas of the World: Priorities for Biodiversity Conservation. BirdLife International. Cambridge, UK. Series No 7, 846 p.

Thiollay JM. 1986. Structure comparée du peuplement avien dans trois sites de forêt primaire en Guyane. La Terre et la Vie, 41 : 59-105.
UICN 2018. Liste rouge l'Union Internationale pour la Conservation de la Nature www.iucn.org, consulté 31/10/2018.

Yaokokoré-Béibro KH. 2001. Avifaune des forêts classées de l'Est de la Côte d'Ivoire : données sur l'écologie des espèces et effet de la déforestation sur les peuplements. Cas des forêts classées de la Béki et de la Bossématié (Abengourou). Thèse de Doctorat de l'Université de Cocody, Abidjan, Côte d'Ivoire, p. 245.

Yaokokoré-Béibro KH. 2010. Les oiseaux. In Atlas de la Biodiversité de l'Afrique de l'Ouest, Tome III, Côte d'Ivoire Konaté S, Kampmann D (eds). BIOTA : Abidjan \& Frankfurt/Main ; 210-216.

Yaokokoré-Béibro KH, Koné SY, Odoukpe SGK, Guèye FM. 2015a. Avifaune d'un milieu marécageux urbain dans la commune de Cocody (Abidjan, Côte d'Ivoire). International Journal of Innovation and Scientific Research, 18(1): 1-10.

Yaokokoré-Béibro KH, Guèye FM, Koné YS, Odoukpe SGK. 2015b. Biodiversité urbaine des Oiseaux dans la Zone humide d'Importance Internationale de GrandBassam (Sud-Est de la Côte d'Ivoire). International Journal of Innovation and Applied Studies, 11(2): 339-349.

Yaokokoré-Béibro KH, Konan EM, Kouadio KP. 2015c. Diversité et abondance des oiseaux de la forêt classée de la Téné, Centre-Ouest Côte d'Ivoire. Journal of Animal \& Sciences, 24: 1-11.

Zhang W, Ricketts TH, Kremen C, Carney K, Swinton SM. 2007. Ecosystem services and dis-services to agriculture. Ecological Economics, 64(2): 253-260. 